Journal of Systems Science and Information

Feb., 2015, Vol. 3, No. 1, pp. 37-47

DOI: $10.1515 /$ jssi-2015-0037

\title{
Managing Pricing of Closed-Loop Supply Chain Under Patent Protection
}

\author{
Xiaogang CAO \\ School of Management, Wuhan Textile University, Wuhan 430073, China; School of Management, \\ Huazhong University of Science and Technology, Wuhan 430074, China \\ E-mail: caoxiaogang1982@hotmail.com \\ Leqi HUA \\ School of Management, Wuhan Textile University, Wuhan 430073, China \\ E-mail: 823487691@qq.com \\ Hui WEN* \\ School of Science, Hubei University of Technology, Wuhan 430068, China \\ E-mail: 351587833@qq.com \\ Yan WU \\ School of Management, Wuhan Textile University, Wuhan 430073, China \\ E-mail: 379395232@qq.com
}

\begin{abstract}
This paper studies the closed-loop supply chain decision problem in which the original manufacturer allows the third-party remanufacturer to remanufacture under patent protection. In the two cycle mode, the authors discuss the pricing decision problem of the original manufacturer, the retailer, and the third-party remanufacturer in centralized and decentralized decision-making cases. The authors obtain the optimal sale price and wholesale price of new products and remanufactured products in two cases, and the optimal recycling rate of used products in centralized decision-making case. Finally, the authors carry out the corresponding numerical simulation of the pricing decision.
\end{abstract}

Keywords closed-loop supply chain; patent protection; pricing decision; Stackelberg game

\section{Introduction}

In the construction machinery market, different grades of products exist for different user groups. Diversified demands make it possible to build the remanufacturing industries, and the rapidly increasing of all kinds of engineering machinery market quantity creates favorable conditions for developing remanufacturing and realizing industrialized economy scale. For reasons

Received March 24, 2014, accepted May 9, 2014

*The corresponding author, E-mail: 351587833@qq.com

Supported by the National Natural Science Foundation of China (71301126), Humanities and Social Sciences Research Project of Ministry of Education in China (11YJC630011), China Postdoctoral Science Foundation (2012M511215, 2013T60723), Humanities and Social Sciences Research Project of Hubei Province Office of Education (2010q054, 2012G080), and University Students' Innovation Entrepreneurship Training Program of Wuhan Textile University (2013) 
of cost or brand, the original manufacturer may not be able to carry out the recycling and remanufacturing activities of used products, so the third-party remanufacturer carries out the remanufacturing activities, which inevitably brings some competitive threats to new products produced by the original manufacture ${ }^{[1]}$. The conflict of interest and intellectual property disputes between the original manufacturer and the remanufacturer is gradually emerging in recent years with the development of remanufacturing industry. This shows that the third-party remanufacturer must be licensed by the original manufacturer when entering the remanufacturing market protected by patents in the case of patent-protected original manufacturer, which helps the original manufacturer acquire competitive advantage. Patented products' remanufacturing issues are related to the authorization of the patent technology, so patent licensing issue must be taken into account in the remanufacturing decision-making, which should also be paid to more attention in remanufacturing research. A rapidly growing stream of literatures on closed-loop supply chain has appeared in recent years. Guide and Van Wassenhovel ${ }^{[2]}$ studied the recycling of used products in a closed-loop supply chain decision-making model which takes into account the quality uncertainty issue of the recycled products. Savaskan et al. ${ }^{[3,4]}$ investigated the pricing policy of the closed-loop supply chain in different recycling structures and channel efficiency. Gu et al. ${ }^{[5]}$ applied game theory to study the optimal policy and efficiency problem of used products' recycling in reverse supply chain system. Vorasayan and Ryans ${ }^{[6]}$ used mathematical programming model to analyze the influence of recycling quality and cost on the optimal decision. Ferguson ${ }^{[7]}$ proposed the closed-loop supply chain coordination problem of the defective products. Qiu et al. ${ }^{[8,9]}$ both investigate the pricing of different form of closedloop supply chain system. Atasu et al. ${ }^{[10]}$ assumed that there exist "green consumers" in the market whose valuation of remanufactured products is lower than new products, and study the decision problem of whether the manufacturer provides the remanufactured products. However, these studies are only focused on new products and new technology licensing, and the remanufacturing patent licensing literature has not yet appeared. When facing competition with the remanufacturing enterprises, the original manufacturers take two main strategies: Remanufacturing and antecedent recovery to compensate the loss of potential profit ${ }^{[11]}$. Xiong et al. ${ }^{[12]}$ established a patent-protected closed-loop supply chain model in which the third-party re-manufacturer is licensed to remanufacture by the original manufacturer, and apply game theory to discuss the optimal decision of the node enterprises in the cases of decentralization and centralization. Oraiopoulos ${ }^{[13]}$ conducted how an OEM's incentives and optimal strategies vis-á-vis the secondary market are shaped contingent on her relative competitive advantage, product characteristics, and consumer preferences. Based on the above literatures, we study the patent-protected closed-loop supply chain decision-making problem in which the original manufacturer licenses the third-party remanufacturing, and analyze the pricing decision of the original manufacturer, the retailer and the third-party remanufacturer in centralized and decentralized decision-making cases in two cycle mode, and we obtain the optimal sale price and wholesale price of new products and remanufactured products in two cases and the optimal recycling rate of used products in centralized decision-making case. The remainder of the paper is as follows: Section 2 illustrates the model framework and some assumptions. The closed-loop supply chain pricing decision under patent protection is studied in Section 3. Section 4 gives 
the numerical analysis to verify our theoretical results, and the paper is concluded in Section 5.

\section{Problem Description and Assumption}

1) Consider a supply chain consisting of the original manufacturer. The retailer and the third-party remanufacturer, in which the original manufacturer is responsible for the production of new products, the third-party remanufacturer is responsible for recycling and remanufacturing of used products, and the retailer is responsible for sales of new products and remanufactured products.

2) Remanufactured products and new products are the same in quality, but consumers have different willingness to pay for the two types of products, and the retailer adopts differential pricing policy for new products and remanufactured products.

3) The total recycling cost is denoted by $C(T)=B T^{2}+A T q_{1 n}$, where $B T^{2}$ represents the scale of investment of the recycling of used products, $T$ denotes the recycling rate of used products and $0<T \leq 1, B$ denotes the scaling parameter, $A$ denotes the unit price of the recycling of used products, and $q_{1 n}$ denotes the market demand for new products in the first phase.

4) $f$ denotes unit patent licensing fee paid to the original manufacturer by the third-party remanufacturer.

5) $Q$ denotes the potential market demand, and $\alpha_{n}$ denotes consumers' willingness to pay for new products, and $\alpha_{r}$ denotes consumers' willingness to pay for remanufactured products, where $\alpha_{n} \in[0, Q], \alpha_{r}=\theta \alpha_{n}$, and $\theta \in(0,1)$ denotes consumers' acceptance degree of remanufactured products relative to new products (referred to as consumers' acceptance degree).

From the above analysis we can obtain that the utility functions of consumers to buy new products and remanufactured products are $u_{n}=\alpha_{n}-P_{n}$ and $u_{r}=\alpha_{r}-P_{r}$, respectively, where $p_{n}$ denotes the price of new products, and $P_{r}$ denotes the price of remanufactured products. $u_{n} \geq 0$ indicates that the consumer is willing to buy new products, and $u_{r} \geq 0 u_{n} \geq u_{r}$ indicates that the consumer is willing to buy remanufactured products. $u_{n}<u_{r}$ indicates that the consumer is more willing to buy new products rather than remanufactured products. Similarly, it indicates that the consumer is more willing to buy remanufactured products rather than new products. According to the calculation results of [14], we obtain the demand function for new products and remanufactured products are

$$
q_{n}=Q-\frac{P_{n}-P_{r}}{1-\theta}
$$

and

$$
q_{r}=\frac{\theta P_{n}-P_{r}}{\theta(1-\theta)}
$$

respectively.

\section{Closed-Loop Supply Chain Decisions with the Third Party Respon- sible for Remanufacturing Under Patent Protection}

The model is divided into two cycles. In the first period, the original manufacturer only produces new products, and the production $\operatorname{cost}$ is $c_{1 n}$, and the products are wholesaled to the 
retailer with wholesale price $w_{1}$. The retailer only sells the new products with sale price $p_{1 n}$ and sale quantity $q_{1 n}$ in the first period; in the second period, the original manufacturer still only produces new products with production $\operatorname{cost} c_{2 n}$ and wholesale price $w_{2 n}$ wholesaled to the retailer. The third-party remanufacturer is responsible for recycling and remanufacturing of the used products in the first period with unit remanufacturing $\operatorname{cost} c_{2 r}$ and unit patent licensing fee $f$ paid to the original manufacturer. Subsequently, the third party remanufacturer wholesales remanufactured products to the retailer with wholesale price $w_{2 r}$, and the retailer simultaneously supplies new products and remanufactured products to the market with sale price $p_{2 n}$ and $p_{2 r}$, respectively.

In the first phase, the original manufacturer only produces new products, so the original manufacturer maximizes its profit function in the first phase

$$
\max \Pi_{M}=\left(W_{1}-C_{1 n}\right) q_{1 n}
$$

and the retailer maximizes its profit function

$$
\max \Pi_{R}=\left(P_{1 n}-w_{1}\right) q_{1 n}
$$

and the market demand function is

$$
q_{1 n}=Q-P_{1 n}
$$

We use Stackelberg game to solve the optimal decisions of the original manufacturer and the retailer in this period, in which the original manufacturer is the leader, and the retailer is the follower. According to [15], we can obtain the optimal wholesale price of new products in the first phase is $W_{1}^{*}=\left(Q+c_{1 n}\right) / 2$, the optimal sale price of new products is $P_{1 n}^{*}=\left(3 Q+c_{1 n}\right) / 4$, the optimal ordering quantity of new products is $q_{1 n}^{*}=\left(Q-c_{1 n}\right) / 4$, the optimal profit of the original manufacturer is $\Pi_{M}^{1 *}=\left(Q-c_{1 n}\right)^{2} / 8$, and the optimal profit of the retailer is $\Pi_{R}^{1 *}=\left(Q-c_{1 n}\right)^{2} / 16$.

In the second period, the third-party remanufacturer is responsible for the recycling and remanufacturing of used products in the first period, therefore, we consider two cases of centralized decision-making and decentralized decision-making in the second period.

\subsection{Centralized Decision-Making Case}

In centralized decision-making model, the original manufacturer, the retailer, and the thirdparty remanufacturer combine to determine the optimal retail price of the products and the recycling price of used products in order to maximize the profit of the entire closed-loop supply chain system, and the wholesale price and patent licensing fee only decide the allocation of the system profit among the members, and do not affect the system's total revenue.

The problem of the closed-loop supply chain system of centralized decision-making is modeled as follows:

$$
\begin{array}{ll}
\max & \Pi\left(P_{2 n}, P_{2 r}, T\right)=\left(P_{2 n}-c_{2 n}\right) q_{2 n}+\left(P_{2 r}-c_{2 r}\right) q_{2 r}-B T^{2}-A T\left(Q-P_{1 n}\right) \\
\text { s.t. } & T\left(Q-P_{1 n}\right)-q_{2 r} \geq 0
\end{array}
$$


Substituting (1) and (2) into (6) we can obtain

$$
\begin{aligned}
& \max \quad \Pi\left(P_{2 n}, P_{2 r}, T\right)=\left(P_{2 n}-c_{2 n}\right)\left(Q-\frac{P_{2 n}-P_{2 r}}{1-\theta}\right) \\
& \quad+\left(P_{2 r}-c_{2 r}\right) \frac{\theta P_{2 n}-P_{2 r}}{\theta(1-\theta)}-B T^{2}-A T\left(Q-P_{1 n}\right) \\
& \text { s.t. } \quad T \frac{Q-c_{1 n}}{4}-\frac{\theta P_{2 n}-P_{2 r}}{\theta(1-\theta)} \geq 0
\end{aligned}
$$

From (8) we can acquire the Hessian matrix of the profit function of the supply chain

$$
\left(\begin{array}{ccc}
\frac{-2}{1-\theta} & \frac{2}{1-\theta} & 0 \\
\frac{2}{1-\theta} & \frac{-2}{\theta(1-\theta)} & 0 \\
0 & 0 & -2 B
\end{array}\right) .
$$

Since $0<\theta<1, B>0$, we know $D_{1}=\frac{-2}{1-\theta}<0, D_{2}=\frac{4-4 \theta}{\theta(1-\theta)}>0, D_{3}=\frac{8 B(\theta-1)}{\theta(1-\theta)}>0$, so the Hessian matrix is negatively definite, and the profit function of the supply chain is concave, and there exists the optimal solution.

Let the Lagrangian function be

$$
\begin{aligned}
L= & \left(P_{2 n}-c_{2 n}\right)\left(Q-\frac{P_{2 n}-P_{2 r}}{1-\theta}\right)+\left(P_{2 r}-c_{2 r}\right) \frac{\theta P_{2 n}-P_{2 r}}{\theta(1-\theta)}-B T^{2} \\
& -A T\left(Q-P_{1 n}\right)+\lambda\left(T \frac{Q-c_{1 n}}{4}-\frac{\theta P_{2 n}-P_{2 r}}{\theta(1-\theta)}\right)
\end{aligned}
$$

We can obtain the following K-K-T conditions:

$$
\begin{aligned}
& -2 P_{n}+2 P_{2 r}+\lambda-c_{2 r}+(1-\theta) Q+c_{2 n}=0 \\
& 2 \theta P_{2 n}-2 P_{2 r}+\lambda+c_{2 r}-\theta c_{2 n}=0 \\
& -8 B T+\lambda\left(Q-c_{1 n}\right)-A\left(Q-c_{1 n}\right)=0 \\
& \lambda\left(T \frac{Q-c_{1 n}}{4}-\frac{\theta P_{2 n}-P_{2 r}}{\theta(1-\theta)}\right)=0
\end{aligned}
$$

Combining (9), (10), (11) and (12) we can obtain

$$
\begin{aligned}
P_{2 n}^{*} & =\frac{Q+C_{2 n}}{2} \\
P_{2 r}^{*} & =\frac{\theta Q+C_{2 r}+\lambda}{2} \\
\lambda^{*} & =\frac{A \theta(1-\theta)\left(Q-c_{1 n}\right)^{2}+16 B\left(\theta c_{2 n}-c_{2 r}\right)}{\theta(1-\theta)\left(Q-c_{1 n}\right)^{2}+16 B} \\
T^{*} & =\frac{2\left(Q-c_{1 n}\right)\left(\theta c_{2 n}-c_{2 r}-A\right)}{\theta(1-\theta)\left(Q-c_{1 n}\right)^{2}+16 B}
\end{aligned}
$$

We can obtain the following result according to the above analysis:

Proposition 1 In centralized decision-making case, the optimal sale price of new products in the second period is

$$
P_{2 n}^{*}=\frac{Q+c_{2 n}}{2}
$$


the optimal sale price of remanufactured products is

$$
P_{2 r}^{*}=\frac{\theta(1-\theta)\left(Q-c_{1 n}\right)^{2}\left(\theta Q+c_{2 r}+A\right)+16 B \theta\left(Q+c_{2 n}\right)}{2 \theta(1-\theta)\left(Q-c_{1 n}\right)^{2}+32 B},
$$

and the optimal recycling rate of used products is

$$
T^{*}=\frac{2\left(Q-c_{1 n}\right)\left(\theta c_{2 n}-c_{2 r}-A\right)}{\theta(1-\theta)\left(Q-c_{1 n}\right)^{2}+16 B}
$$

\subsection{Decentralized Decision-Making Case}

The original manufacturer maximizes its profit function

$$
\max \Pi_{M}\left(w_{2 n}\right)=\left(w_{2 n}-c_{2 n}\right) q_{2 n}+f q_{2 r}=\left(w_{2 n}-c_{2 n}\right)\left(Q-\frac{P_{2 n}-P_{2 r}}{1-\theta}\right)+f \frac{\theta P_{2 n}-P_{2 r}}{\theta(1-\theta)}
$$

The retailer maximizes its profit function

$$
\begin{aligned}
\max \Pi_{R}\left(P_{2 n}, P_{2 r}\right) & =\left(P_{2 n}-W_{2 n}\right) q_{2 n}+\left(P_{2 r}-W_{2 r}\right) q_{2 r} \\
& =\left(P_{2 n}-w_{2 n}\right)\left(Q-\frac{p_{2 n}-p_{2 r}}{1-\theta}\right)+\left(P_{2 r}-w_{2 r}\right) \frac{\theta P_{2 n}-P_{2 r}}{\theta(1-\theta)}
\end{aligned}
$$

The third party remanufacturer maximizes its profit function

$$
\begin{aligned}
& \max \quad \Pi_{T}\left(W_{2 r}\right)=\left(W_{2 r}-c_{2 r}-f\right) q_{2 r}-B T^{2}-A T\left(Q-P_{1 n}\right) \\
& =\left(W_{2 r}-c_{2 r}-f\right) \frac{\theta P_{2 n}-P_{2 r}}{\theta(1-\theta)}-B T^{2}-A T\left(Q-P_{1 n}\right) \\
& \text { s.t. } \quad T \frac{Q-c_{1 n}}{4}-\frac{\theta_{2 n}-P_{2 r}}{\theta(1-\theta)} \geq 0
\end{aligned}
$$

In this paper, we use the Stackelberg game to solve the optimal decision of the original manufacturer, the retailer and the third-party remanufacturer in this mode. Firstly, the original manufacturer is seen as the leader, and the retailer is seen as the follower. The original manufacturer determines the wholesale price of new products $W_{2 n}$ to maximize his revenue, and the retailer decides the retail price of new products according to the market demand and wholesale price of new products; secondly, the third-party remanufacturer is seen as the leader, and the retailer is seen as the follower. The third-party remanufacturer firstly determines the wholesale price $W_{2 r}$ of remanufactured products, and then the retailer decides the retail price of remanufactured products according to the market demand and wholesale price of remanufactured products $P_{2 r}$.

Taking partial derivative of the profit function of the retailer with respect to $P_{2 n}$ and letting it equal to zero, we can obtain

$$
P_{2 n}=P_{2 r}+\frac{W_{2 n}-W_{2 r}+(1-\theta) Q}{2}
$$

Substituting (20) into (18) and taking partial derivative of (18) with respect to $W_{2 n}$, and then letting it equal to zero, we can obtain

$$
W_{2 n}=\frac{Q(1-\theta)+c_{2 n}+f+W_{2 r}}{2}
$$


Taking partial derivative of the function of the retailer with respect to $P_{2 r}$ and letting it equal to zero, we can obtain

$$
P_{2 r}=\frac{2 \theta P_{2 n}-\theta W_{2 n}+W_{2 r}}{2}
$$

Substituting (22) into (20) and taking partial derivative of it with respect to, and letting it equal to zero, we can obtain

$$
W_{2 r}=\frac{\theta W_{2 n}+c_{2 r}+f}{2}
$$

so the simultaneous solution of $(20),(21),(22)$, and (23) is

$$
\begin{aligned}
W_{2 n}^{* *} & =\frac{Q(1-\theta)+c_{2 n}+c_{2 r}+f}{2-\theta} \\
W_{2 r}^{* *} & =\frac{Q \theta(1-\theta)+\theta c_{2 n}+2 c_{2 r}+2 f}{4-2 \theta} \\
P_{2 n}^{* *} & =\frac{Q(3-2 \theta)+c_{2 n}+c_{2 r}+f}{4-2 \theta} \\
P_{2 r}^{* *} & =\frac{\theta c_{2 n}+2 c_{2 r}+2 f+\theta Q(5-3 \theta)}{8-4 \theta}
\end{aligned}
$$

We can obtain the following result according to the above analysis.

Proposition 2 In the second period, the optimal sale price of new products in decentralized decision-making case is

$$
P_{2 n}^{* *}=\frac{Q(3-2 \theta)+c_{2 n}+c_{2 r}+f}{4-2 \theta}
$$

the optimal sale price of remanufactured products in decentralized decision-making case is

$$
P_{2 r}^{* *}=\frac{\theta c_{2 n}+2 c_{2 r}+2 f+\theta Q(5-3 \theta)}{8-4 \theta},
$$

the optimal wholesale price of new products in decentralized decision-making case is

$$
W_{2 n}^{* *}=\frac{Q(1-\theta)+c_{2 n}+c_{2 r}+f}{2-\theta},
$$

the optimal sale price of remanufactured products in decentralized decision-making case is

$$
W_{2 r}^{* *}=\frac{Q \theta(1-\theta)+\theta c_{2 n}+2 c_{2 r}+2 f}{4-2 \theta} .
$$

Since $\frac{\partial P_{2 n}^{* *}}{\partial \theta}=\frac{c_{2 n}+c_{2 r}+f-Q}{(4-2 \theta)^{2}}, \frac{\partial W_{2 n}^{* *}}{\partial \theta}=\frac{c_{2 n}+c_{2 r}+f-Q}{(2-\theta)^{2}}$, we can obtain the following result.

Corollary 1 In the second period, if $c_{2 n}+c_{2 r}+f \geq Q$, then the optimal sale price and wholesale price of new products in decentralized decision-making case are increasing with respect to consumers' acceptance degree to remanufactured products; if $c_{2 n}+c_{2 r}+f \leq Q$, then the optimal sale price and wholesale price of new products in decentralized decision-making case are decreasing with respect to consumers' acceptance degree to remanufactured products. 


\section{Numerical Simulation}

In this paper, we will carry out the numerical simulation analysis of the model, and in the process of simulation, we refer to [16] in parameter settings. Using Mathematica 7.01 programming, the following results can be obtained in the computer.

Letting $Q=1000, c_{1 n}=600, c_{2 n}=600, c_{2 r}=300, A=150, B=10000, f=50$ (we are aware that $c_{2 n}+c_{2 r}+f \leq Q$, which accords with the second case in Corollary 1 ), and making consumers' acceptance degree $\theta$ varies in $[0.65,0.95]$. We will compute the optimal sale price of remanufactured products and recycling rate of used products in centralized decision-making case and the optimal wholesale price and sale price of new products in decentralized decisionmaking case. In the following figures, we all use horizontal ordinate $x$ to denote consumers' acceptance degree. In Figure 1, longitudinal ordinate $y_{1}$ denotes the optimal sale price of remanufactured products in centralized decision-making case; in Figure 2, longitudinal ordinate $y_{2}$ denotes the optimal recycling rate of used products in centralized decision-making case; in Figure 3 , longitudinal ordinate $y_{3}$ denotes the optimal sale price of new products in decentralized decision-making case; in Figure 4, longitudinal ordinate $y_{4}$ denotes the optimal sale price of remanufactured products in decentralized decision-making case; in Figure 5, longitudinal ordinate $y_{5}$ denotes the optimal wholesale price of new products in decentralized decision-making case; in Figure 6, longitudinal ordinate $y_{6}$ denotes the optimal wholesale price of remanufactured products in decentralized decision-making case.

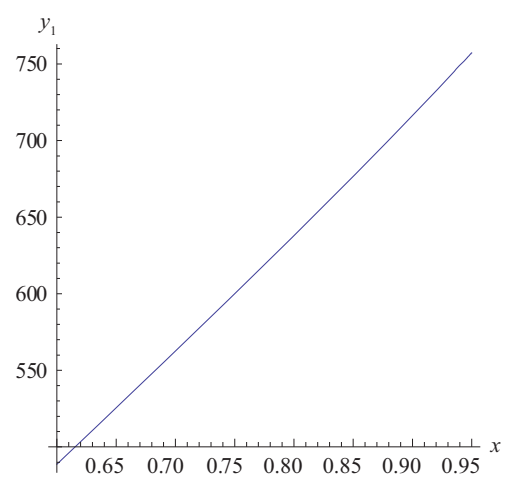

Figure 1 The optimal sale price of remanufactured products in centralized decision-making case

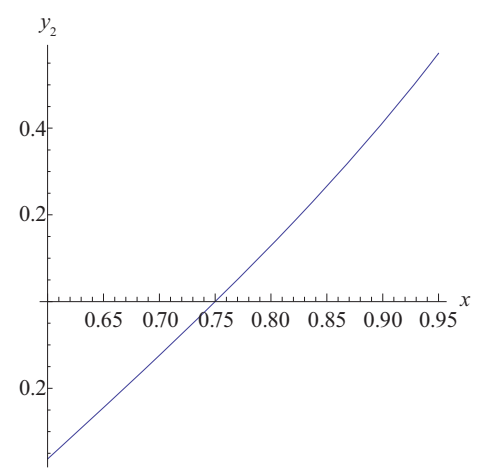

Figure 2 The optimal recycling price of used products in centralized decision-making case 


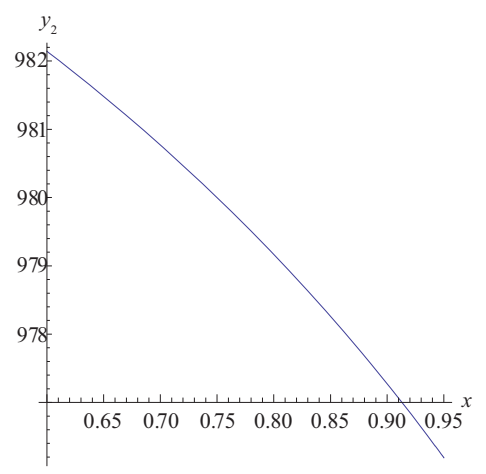

Figure 3 The optimal sale price of new products in decentralized decision-making case

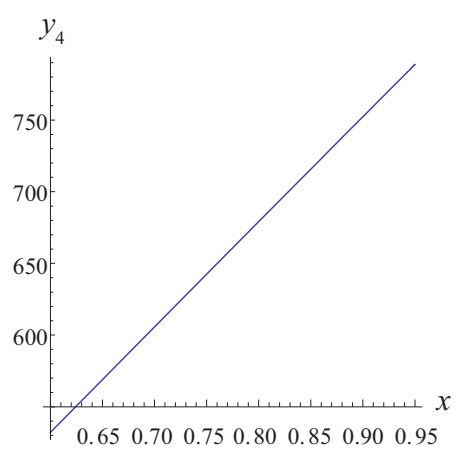

Figure 4 The optimal sale price of remanufactured products in decentralized decision-making case

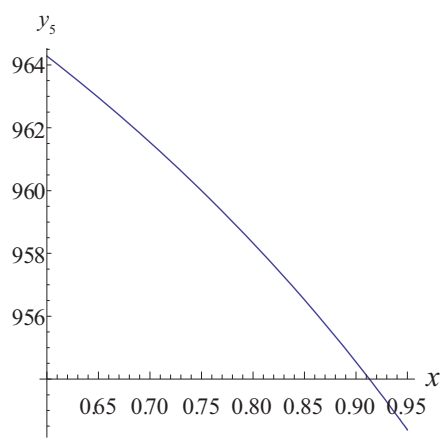

Figure 5 The optimal wholesale price of new products in decentralized decision-making case

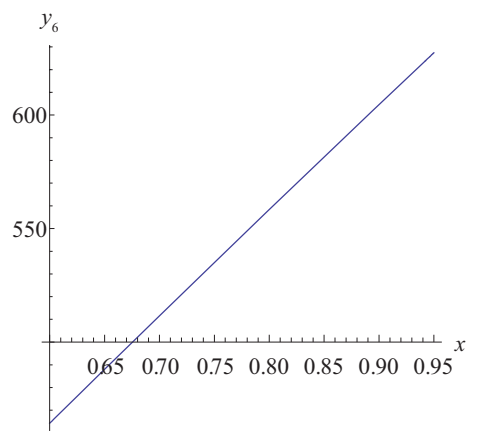

Figure 6 The optimal wholesale price of remanufactured products in decentralized decision-making case 
From Figure 1 we can find that in the second stage, the optimal sale price of remanufactured products is increasing with respect to consumers' acceptance degree to remanufactured products in centralized decision-making case, which is because the increase of consumers' acceptance degree to remanufactured products leads to the increase of the demand of remanufactured products. As the remanufacturing cost is less than that of new products, raising the sale price of remanufactured products will increase the whole supply chain's profit from the point of the entire supply chain. From Figure 2 we can find that in the second stage, the optimal recycling rate of used products is increasing with respect to consumers' acceptance degree to remanufactured products in centralized decision-making case, which is because the increase of consumers' acceptance degree to remanufactured products leads to the increase of the demand of remanufactured products, and the increase of recycling rate of used products will increase the quantity of remanufactured products. From Figure 3 we can find that in the second stage, the optimal sale price of new products is decreasing with respect to consumers' acceptance degree to remanufactured products in decentralized decision-making case, which accords with the second case in Corollary 1. This is because the increase of consumers' acceptance degree to remanufactured products leads to the increase of the demand of remanufactured products. As the potential market demand is bigger than the sum of manufacturing cost, remanufacturing cost, and patent licensing fee, which results in that the retailer raises the demand of new products through decreasing the sale price of new products. From Figure 4 we can find that in the second stage, the optimal sale price of remanufactured products is increasing with respect to consumers' acceptance degree to remanufactured products in decentralized decision-making case, which is because the increase of consumers' acceptance degree to remanufactured products leads to the increase of the demand of remanufactured products. So raising the sale price of remanufactured products will increase the retailer's profit. From Figure 5 we can find that in the second stage, the optimal wholesale price of new products is decreasing with respect to consumers' acceptance degree to remanufactured products in decentralized decision-making case, which is because the increase of consumers' acceptance degree to remanufactured products leads to the decrease of the demand of new products, so the original manufacturer can increase the retailer's ordering quantity of new products through decreasing the wholesale price of new products, which will also increase the original manufacturer's profit. From Figure 6 we can find that in the second stage, the optimal wholesale price of remanufactured products is increasing with respect to consumers' acceptance degree to remanufactured products in decentralized decision-making case, which is because the increase of consumers' acceptance degree to remanufactured products leads to the increase of the sale price of remanufactured products, so the third-party remanufacturer can increase his profit through increasing the wholesale price of remanufactured products.

\section{Concluding Remarks}

In this paper, we investigate the closed-loop supply chain decision problem in which the original manufacturer allows the third-party remanufacturer to remanufacture under patent protection. In the two cycle modes, we discuss the pricing decision problem of the original manufacturer, the retailer, and the third-party remanufacturer in two cases of centralized and 
decentralized decision-making. We obtain the optimal retail price and wholesale price of new products and remanufactured products, and the optimal recycling rate of used products in two cases, and simulate the corresponding pricing decision numerically. The results indicate that in the second stage, the optimal sale price of remanufactured products and recycling rate of used products in centralized decision-making case are both increasing with respect to consumers' acceptance degree to remanufactured products. The optimal retail price and wholesale price of new products in decentralized decision-making case are decreasing with respect to consumers' acceptance degree to remanufactured products, and the optimal retail price and wholesale price of remanufactured products in decentralized decision-making case are increasing with respect to consumers' acceptance degree to remanufactured products.

\section{References}

[1] Yin X T. Explication to the newly-adapted patent law. Beijing: Intellectual Property Press, 2005 (in Chinese).

[2] Guide V D R, Van Wassenhovel N. Managing product returns for remanufacturing. Production and Operations Management, 2001, 10(2): 142-155.

[3] Savaskan R C, Bhattachary A S, Van Wassenhovel N. Closed-loop supply chain models with product remanufacturing. Management Science, 2004, 50(2): 239-252.

[4] Huang Z Q, Da Q L. Study on efficiency of serial supply chains with remanufacturing. Journal of Management Sciences in China, 2006, 9(4): 51-56 (in Chinese).

[5] Gu Q L, Gao T G, Shi L S. Price decision analysis for reverse supply chain based on game theory. Systems Engineering - Theory \& Practice, 2005, 25(3): 20-25 (in Chinese).

[6] Vorasayan J, Ryans M. Optimal price and quantity of refurbished products. Production and Operations Management, 2006, 15(3): 369-383.

[7] Ferguson M, Guide V D R, Souzag C. Supply chain coordination for false failure returns. Manufacturing and Service Operations Management, 2006, 8(4): 376-393.

[8] Qiu R Z, Huang X Y. Coordination model for closed-loop supply chain with product recycling. Journal of Northeastern University (Natural Science Edition), 2007, 53(6): 883-886 (in Chinese).

[9] Ge J Y, Huang P Q, Wang Z P. Closed-loop supply chain coordination research based on game theory. Systems Engineering — Theory Methodology Applications, 2007, 16(5): 549-552 (in Chinese).

[10] Atasu A, Sarvery M, Van Wassenhove L N. Remanufacturing as a marketing strategy. Management Science, 2008, 54(10): 1-16.

[11] Bakal I S, Akcali E. Effects of random yield in remanufacturing with price-sensitive supply and demand. Production and Operations Management, 2006, 15(3): 407-420.

[12] Xiong Z K, Shen C R, Peng Z Q. Closed-loop supply chain coordination research with remanufacturing under patent protection. Journal of Management Sciences in China, 2011, 14(6): 76-85 (in Chinese).

[13] Oraiopoulos N, Ferguson M E, Toktay L B. Relicensing as a secondary market strategy. Management Science, 2012, 58(5): 1022-1037.

[14] Xu F, Sheng Z H, Chen G H. The remanufactured products pricing strategy in a heterogeneous market. Chinese Journal of Management Science, 2008, 16(6): 130-136 (in Chinese).

[15] Ferguson M, Toktay L B. The effect of competition on recovery strategies. Production and Operations Management, 2006, 15(3): 351-368.

[16] Xiong Z K, Wang K, Xiong Y, Wang X P. Closed-loop supply chain considering that the distributor engages in remanufacturing. Journal of Systems Engineering, 2011, 26(6): 792-800 (in Chinese). 